\title{
Patologías dentales en incisivos, caninos y primer premolar en caballos chilenos adultos
}

\author{
Dental pathologies in incisors, canines and first premolar in adult Chilean horses \\ L Muñoz*, F Vidal, O Sepúlveda, O Ortiz, C Rehhof \\ Departamento de Ciencias Clínicas, Facultad de Ciencias Veterinarias, \\ Universidad de Concepción, Concepción, Chile.
}

\begin{abstract}
SUMMARY
Dental pathologies have reached a higher veterinary clinical relevance, increasing the number of consultations, treatments and preventive proceedings. In Chilean horses, the information of dental pathologies is inexistent making the research and the data collection in this breed interesting. One hundred horses that came from the Asociación de Rodeo de Concepción, were analyzed between June and September 2007. The materials used were nosetwitch, flashlight, dental probe and intra-oral mirror. The visit started with an interview, followed by an anamnesis and clinical examination. The findings were all registered in a dental examination record. Eighty eight horses presented some kind of dental pathologies (88\%). The most frequent pathologies were fractures (46\%), $\operatorname{tartar}(33 \%)$ and braquignatia (32\%), all of these located in the incisors ('01-'03). Seventy seven horses (55 males and 22 females) had one or more canines (77\%); out of those, 38 (49.35\%) presented pathologies in canines and most commonly, blind canines $(24.67 \%)$. There were 16 horses (13 males and 3 females) with one or more of the first premolar (wolf tooth) (16\%), none of them mandibular. Only one pathology was found in the first premolar, corresponding to a periodontal disease. Statistical differences linked to sex were found the slant mouth in incisors, and for blind canines.
\end{abstract}

Palabras clave: caballo chileno, patologías dentales, odontología equina.

Key words: Chilean horse, dental pathologies, equine odontology.

\section{INTRODUCCIÓN}

La domesticación ha tenido como consecuencia la selección dirigida de caballos que hace más factible la aparición de patologías hereditarias. Asociado a esto, los cambios en el manejo alimenticio han provocado alteración en los modelos de desgaste natural de los dientes que han causado diversas patologías dentales en equinos (Arenas 2003). Las patologías dentales de acuerdo a su origen pueden ser del desarrollo, dentro de las cuales se encuentran braquignatia, prognatismo o retención de deciduos, y adquiridas, como fracturas, caries, enfermedad periodontal o tártaro (Dixon y Dacre 2005). A nivel mundial, en los últimos años se han realizado diversos estudios de prevalencia de patologías dentales en equinos, que han permitido establecer la importancia de éstos en su bienestar y rendimiento. En Chile se han efectuado algunos estudios al respecto; sin embargo, ninguno en el caballo chileno; de ahí la importancia de este estudio, cuyo objetivo fue identificar, cuantificar y comparar las patologías dentales en incisivos, caninos y primer premolar en caballos chilenos adultos y establecer si existían diferencias asociadas al sexo.

Aceptado: 02.09.2009.

* Casilla 160-C, correo 3, Concepción, Chile; lismunoz@udec.cl

\section{MATERIAL Y MÉTODOS}

Entre junio y septiembre de 2007 se examinaron 100 caballos chilenos, adultos de 5 a 24 años de edad, inscritos en la Asociación de Rodeo de Concepción, residentes en criaderos y clubes de Rodeo Chileno de la Provincia de Concepción, VIII Región del Bío-Bío.

El examen dental de incisivos, caninos y primer premolar se realizó siguiendo el procedimiento utilizado por Easley (2002). En algunos caballos se utilizó sonda odontológica, espejo o palpación manual para realizar el diagnóstico. Para identificar las piezas dentales se utilizó la nomenclatura de Triadan modificada (Lowder 1998). Las patologías dentales, datos de identificación del caballo como nombre del caballo, nombre del propietario, número de registro en la Sociedad Nacional de Agricultura, nombre del criadero o club al que pertenecía, sexo y edad fueron anotados en una ficha odontológica individual. Los equinos, de acuerdo al sexo se dividieron en dos grupos: machos $(n=61)$ y hembras $(n=39)$ y de acuerdo a la edad en dos grupos: de 5 a 12 años $(n=74)$ y mayores a 12 años $(\mathrm{n}=26)$.

\section{ANÁLISIS ESTADÍSTICO}

Para el análisis de los datos y cálculo de frecuencias se usó la planilla electrónica Microsoft ${ }^{\circledR}$ Excel Windows. Además, se analizó si existían diferencias dentro cada patología asociadas al sexo o grupo etario, así como también se compararon las diferencias existentes de una 
misma patología a una misma edad en diferente sexo, para lo que se utilizó la Prueba Exacta de Fisher con un nivel de significancia de $\mathrm{P}<0,05$.

\section{RESULTADOS Y DISCUSIÓN}

Dixon (2003) señala que las patologías en incisivos, caninos y primer premolar son poco comunes en comparación con problemas en los dientes de mejilla; esto está en desacuerdo con los resultados obtenidos en este estudio, en donde, de los 100 caballos examinados, 88 presentaron una o más patologías dentales en una o más piezas, porcentaje similar a lo reportado en otros estudios realizados en Chile, como el de Espinosa (2007), quien observó que el 99,02\% de las 103 cabezas que examinó en mataderos de la Provincia de Nuble o Esturillo (2008) que reportó que el 89,6\% de 106 caballos fina sangre inglés del Club Hípico de Concepción presentaban patologías dentales, respectivamente. Lamentablemente en otros trabajos no se señala el porcentaje total de patologías en estos tres segmentos estudiados.

El $84 \%$ de los caballos presentaba alguna patología de los incisivos en donde se encontraron 146 afecciones, de las cuales 38 correspondían a patologías del desarrollo $(26,02 \%)$ y 108 a adquiridas $(73,97 \%)$, resultado similar a lo reportado por Espinosa (2007) y Esturillo (2008), pero superior al 50\% obtenido por Arenas (2003) quien muestreó 240 cabezas en mataderos de la Región Metropolitana y Estrada (2006) que muestreó 50 cabezas en un matadero de la Provincia de Concepción, ambos estudios también en Chile y al 28\% reportado por Chabchoub y col (2005); sin embargo, en este último estudio al igual que en la mayoría de las publicaciones extranjeras no se consideró la presencia de tártaro como patología, lo que obviamente reduce el porcentaje de éstos. En el cuadro 1 se presenta la frecuencia de las patologías de incisivos diferenciados por patología y sexo. Las piezas más afectadas tanto en machos como en hembras fueron la 101, 102, 201 y 202.

La patología más frecuentemente encontrada en los incisivos fue fractura, con un $46 \%$. Este resultado es superior a lo reportado por otros autores que obtuvieron rangos de 2,47 a 38,46\% (Dixon y col 1999, Arenas 2003, Estrada 2006, Espinosa 2007, Pimentel y col 2007, Esturillo 2008). Las fracturas más frecuentes fueron de tamaño pequeño, menores a $5 \mathrm{~mm}$, provocadas probablemente al morder superficies duras durante la estabulación como ocurre en mordedores de madera o tragadores de aire (Caldwell 2006ª); esto quizás explique la diferencia con otros trabajos en que tal vez sólo se consideraron fracturas de mayor tamaño provocadas generalmente por traumatismos importantes como coces (Dixon 2003). La segunda patología de mayor relevancia en los incisivos fue tártaro con un 33\%, similar al $29 \%$ reportado por Brigham y Duncanson (2000), quienes examinaron 100 caballos en el Reino Unido, pero menor a lo reportado por otros autores que obtuvieron frecuencias entre 50 y $100 \%$ en estudios realizados en Chile (Arenas 2003, Estrada 2006, Espinosa 2007, Esturillo 2008); tal vez este bajo porcentaje obedece al manejo y tipo de alimentación del caballo chileno en la zona Central de Chile, en donde muchas veces los caballos tienen acceso al pastoreo, reduciendo de esta manera los factores predisponentes de la aparición de tártaro como son la alimentación con heno o granos secos y la disminución de movimientos masticatorios (Klugh 2006). Publicaciones extranjeras no consideran importante la presencia de tártaro en el caballo, por lo que no lo reportan, aun cuando provoca gingivitis y eventualmente puede favorecer el desarrollo de enfermedad

Cuadro 1. Frecuencia de patologías en incisivos en caballos chilenos. Frequency of incisor pathologies in Chilean horses.

\begin{tabular}{lccccc}
\hline Patología & $\begin{array}{c}\text { Total de } \\
\text { caballos afectados }\end{array}$ & $\begin{array}{c}\text { Total machos } \\
(\mathrm{n}=61)\end{array}$ & $\begin{array}{c}\text { Porcentaje } \\
(\%)\end{array}$ & $\begin{array}{c}\text { Total hembras } \\
(\mathrm{n}=39)\end{array}$ & $\begin{array}{c}\text { Porcentaje } \\
(\%)\end{array}$ \\
\hline Fractura & 46 & 30 & 49,18 & 16 & 41,02 \\
Tártaro & 33 & 18 & 29,51 & 15 & 38,46 \\
Braquignatia & 32 & 22 & 36,06 & 10 & 25,64 \\
Enf. periodontal & 11 & 6 & 9,83 & 5 & 12,82 \\
Sonrisa & 9 & 6 & 9,83 & 3 & 7,69 \\
Boca en declive & 6 & $6^{\mathrm{a}}$ & 9,83 & 1 & 0 \\
Diastema & 3 & 2 & 3,27 & 0 & 0 \\
Retención & 2 & 2 & 3,27 & 0 & 0 \\
Pérdida de pieza & 2 & 2 & 3,27 & 1,64 & 0 \\
Oligodontia & 1 & 1 & 1,64 & 0 & 0 \\
Extrusión & 1 & 1 & & 0 \\
\hline
\end{tabular}

$\mathrm{N}=100$.

Las letras a y b indican diferencia estadística $(\mathrm{P}<0,05)$. 
periodontal (Johnson y Porter 2006, Klugh 2006). De las anormalidades del desarrollo de los incisivos, la que más se presentó fue braquignatia con $32 \%$; ésta es hereditaria y se produce por acortamiento de la mandíbula o bien por sobrecrecimiento de la maxila (Dixon 2003, Dixon y Dacre 2005). El porcentaje obtenido fue muy similar a lo reportado por Peters y col (2006) y Esturillo (2008), pero mayor a lo reportado por otros autores con rangos de 1 a 23,52\% (Dixon y col 1999, Brigham y Duncanson 2000, Vlaminck y col 2001, Núñez y col 2004, Chabchoub y col 2005, Dacre 2006, Espinosa 2007, Pimentel y col 2007). De los 32 casos de braquignatia, siete eran leves, lo que significa que los incisivos maxilares no sobrepasan totalmente la línea de los incisivos mandibulares; los otros 25 presentaban una braquignatia total, en que los incisivos maxilares sobrepasan completamente la línea de los mandibulares, según la definición realizada por Peters y col (2006). Este alto porcentaje se podría explicar por la consanguinidad existente en el caballo chileno al ser una raza con registros cerrados, lo que también ocurre en los fina sangre de carrera.

Enfermedad periodontal se presentó en el 11\% de los caballos examinados, ésta causada principalmente por acumulo de alimento entre los dientes que produce alteración de la flora normal de la boca y que se manifiesta con dolor, enrojecimiento e inflamación de la encía, regresión gingival y en casos crónicos y severos pérdida de la estabilidad del diente (Tremaine 2004, Klugh 2006). Este porcentaje es bastante alto al compararlo con lo informado por Dixon y col (1999), quienes reportaron sólo tres casos de 400 caballos referidos a una clínica en Escocia. Otros estudios señalan prevalencias mayores de enfermedad periodontal de entre 24 y 42,25\% (Arenas 2003, Estrada 2006), pero lamentablemente en ellos se incluye el segmento dientes de mejilla, lo que no permite discutir estos resultados. La sonrisa es el sobrecrecimiento de los incisivos superiores por falta de desgaste como consecuencia de la braquignatia (Johnson y Porter 2006), el porcentaje obtenido en el presente estudio es similar al 9,3\% reportado por Peters y col (2006), pero muy inferior al $24 \%$ reportado por Estrada (2006). La boca en declive es una manifestación de la falta de uso de las piezas dentales de un lado causado por dolor crónico generalmente consecuencia de trauma en los incisivos, presencia de caries o sobrecrecimiento en los dientes de mejilla (Johnson y Porter 2006) o problemas en la articulación temporomandibular (Johnson y Porter 2006, Schumacher 2006), que se presentó en el 6\% de los caballos examinados, resultado igual al obtenido por Estrada (2006) y levemente mayor a lo encontrado por Peters y col (2006). En nuestro estudio la patología se presentó sólo en machos, lo que es estadísticamente significativo $(\mathrm{P}=0,046)$ y no había sido reportado en otros estudios; esto aparentemente no tiene una explicación, sin embargo, se podrían hacer estudios futuros en busca de posibles factores predisponentes como alimentación o comportamiento. Diastema se define como el espacio anormal que se genera entre dos dientes adyacentes, generalmente asociado a la retención de incisivos deciduos, a fractura parcial o pérdida de piezas dentales, pero también al desgaste normal de las piezas dentales en individuos mayores a 20 años (Dixon y Dacre 2005). Esta patología se encontró en el 3\% de los caballos examinados, porcentaje menor a lo reportado en otros estudios con porcentajes de entre 8 y $12 \%$ (Arenas 2006, Espinosa 2007), lo que podemos relacionar con el bajo porcentaje de retención de incisivos deciduos que se observó en el presente estudio, que alcanzó sólo al 2\%, mientras que en el estudio de Estrada (2006) la retención alcanzó al 10\%. En otros estudios se reportan retenciones de entre 1,25 a 7\% (Dixon y col 1999, Brighman y Duncanson 2000). La pérdida de incisivos también fue baja con un $2 \%$, ellas habitualmente son consecuencia de fracturas de dientes definitivos o caída de éstos como consecuencia de enfermedad periodontal. El porcentaje es similar al reportado por Chabchoub y col (2005) y Espinosa (2007). La oligodontia, que se define como la ausencia de desarrollo de un diente (Dixon y Dacre 2005), puede ser de origen congénito o bien adquirida por el traumatismo del brote dental (Dacre 2006, Easley 2006, Johnson y Porter 2006). En el presente estudio se encontró sólo un caballo con este problema (1\%), similar a lo reportado por Estrada (2006) y Espinosa (2007), y coincidente con lo señalado por Razman y col (2001) quienes describieron un caso de oligodontia asociada a prognatismo y displasia dental. El paso o extrusión dental, definido como el sobrecrecimiento de una pieza dental por falta de desgaste como consecuencia de la ausencia de contacto con un diente opuesto (Dixon 2003, Dixon y Dacre 2005), se encontró en un solo equino; esto no es reportado en otros estudios, probablemente porque los caballos examinados habían sido sometidos a limado dental. En nuestro estudio no se observaron algunas patologías descritas en otros estudios como prognatismo (Núñez y col 2004, Espinosa 2007, Pimentel y col 2007), incisivos supernumerarios (Dixon y col 1999, Estrada 2006), tumores dentales (Dixon y col 1999, Estrada 2006), convexidad de la superficie oclusal de los incisivos inferiores (Peters y col 2006, Espinosa 2007) o infección apical (Dixon y col 1999).

De los 100 caballos, 77 tenían uno o más caninos, porcentaje mayor al reportado en otros estudios con frecuencias de entre 35 y $52 \%$ (Arenas 2003, Estrada 2006, Espinosa 2007, Esturillo 2008). El alto porcentaje de presencia de caninos al compararlo con el trabajo de Esturillo (2008) se puede explicar porque en él incluyó principalmente caballos menores a 6 años, lo que implica que en muchos de ellos no habían erupcionado los caninos, ya que los caninos erupcionan entre los 3 y medio y 5 años (Caldwell 2006 $6^{\mathrm{b}}$, Linkous 2006). De los caballos con caninos, 55 eran machos $(90,01 \%)$ y 22 hembras $(56,44 \%)$, en el caso de los machos la presencia de caninos fue alta, pero dentro del rango reportado por otros autores que indican presencia de caninos en 83 a 100\% de los casos; sin embargo, en el caso de las hembras este porcentaje 
es muy superior a lo observado en otros trabajos que reportan presencia de caninos entre el 8 y $28 \%$ en éstas (Arenas 2003, Caldwell 2006 ${ }^{\mathrm{b}}$, Estrada 2006, Espinosa 2007, Esturillo 2008), lo que tal vez sea una característica racial. De los 77 caballos que poseían caninos, 57 de ellos tenían todas las piezas y 20 tenían uno a tres caninos. De éstos, 38 caballos presentaban patologías (49,35\%), 22 machos $(28,57 \%)$ y 16 hembras $(20,78 \%)$, muy por debajo del $100 \%$ reportado por Espinosa (2007), pero muy superior al $1,25 \%$ reportado por Dixon y col (1999) quien en su estudio sólo consideró los casos que eran referidos a su clínica con problemas específicos, es decir, sólo 5 de los 400 caballos. En el cuadro 2 se muestra la frecuencia de patologías de caninos por patología y sexo. Los caninos ciegos fueron la patología más frecuente en esta pieza, con un $24,67 \%$. Frecuencia mayor a lo reportado por otros autores que obtuvieron frecuencias de 0,025 a 14,08\% (Dixon y col 1999, Brigham y Duncanson 2000, Arenas 2003, Estrada 2006). En este trabajo la mayoría de los caninos ciegos fueron encontrados en hembras $(72,73 \%)$ y sólo $5,45 \%$ en machos $(\mathrm{P}=0,00001)$. Esta diferencia tiene directa relación con la mayor presencia de caninos entre machos y hembras, ya que en éstas, cuando está presente el canino, en general es de menor tamaño y/o se presenta formando una especie de nódulo o botón, lo que corresponde a canino ciego (Dixon y Dacre 2005, Caldwell 2006 ${ }^{b}$ ). La segunda patología de mayor frecuencia en caninos fue el tártaro con un $15,58 \%$, porcentaje muy inferior a lo encontrado por otros autores que reportaron frecuencias de 36 a 100\% (Brigham y Duncanson 2000, Estrada 2006, Espinosa 2007, Esturillo 2008). Tal vez, debido a los mismos motivos indicados anteriormente para los incisivos, ya que dentro de los factores predisponentes está la mala masticación y la falta de forraje en la ración (Easley 2004). La presencia de tártaro o sarro dental es muy común en los caninos (Klugh 2006, Linkous 2006), sin embargo, pese a que en general se asocia a gingivitis, rara vez produce pérdida de esta pieza (Kugh 2006). Las piezas más afectadas con tártaro fueron las mandibulares
(304 y 404) con un 85\% del total de caninos afectados, resultado similar a lo reportado por Arenas (2003) y Esturillo (2008). Lo anterior, difiere de lo encontrado por Estrada (2006) quien reportó que los caninos afectados con tártaro se repartían uniformemente entre las cuatro piezas. Otro de los problemas detectados en caninos fue crecimiento excesivo en el 6,49\% de los casos, dentro del rango de lo reportado por otros autores quienes observaron frecuencias de 1,67 a 12\% (Brigham y Duncanson 2000, Arenas 2003), lo que puede provocar daño en la mucosa bucal o lengua (Linkous 2006), así como también poner en riesgo a una persona al momento de colocar el freno o manipular la lengua (Caldwell 2006 ${ }^{\text {}}$ ). Además, se detectó un 5,19\% de enfermedad periodontal, lo cual es raro según lo señalado por Klugh (2006), y que se manifestó en estos casos con gingivitis; sin embargo, como la principal causa de enfermedad periodontal en el caballo es el acúmulo de alimento, situación poco habitual en el canino, esta enfermedad generalmente no evoluciona hacia la caída del diente, salvo cuando existe un canino desplazado o retenido (Caldwell 2006 ${ }^{\text {b }}$. Fracturas de caninos provocadas generalmente en forma iatrogénica asociada a la colocación del freno se observaron en dos casos $(2,59 \%)$, lo cual está dentro de lo observado por otros autores que reportan incidencias bastante bajas y en su mayoría asociadas a esta misma causa (Dixon y col 1999, Caldwell $2006^{\text {b }}$ ) o como consecuencia de una coz o de morder una superficie dura (Caldwell 2006 ${ }^{\mathrm{b}}$ ). Un canino desplazado $(1,3 \%)$ hacia mesial se encontró en el presente estudio, porcentaje muy similar a lo reportado por Dixon y col (1999) y Arenas (2003), situación que puede favorecer la acumulación de alimentos en el diastema que se crea entre el canino y el incisivo extremo. No se encontraron caninos supernumerarios, patología reportada por Dixon y col (1999) en uno de los 400 caballos examinados.

El primer premolar o "diente de lobo" ( '05) se presentó en el $16 \%$ de los caballos muestreados, porcentaje dentro de lo observado por otros autores, que reportaron frecuencias de 10 a $90 \%$ (Brigham y Duncanson 2000, Scrutchfield 2002,

Cuadro 2. Frecuencia de patologías en caninos en caballos chilenos. Frequency of canine pathologies in Chilean horses.

\begin{tabular}{|c|c|c|c|c|c|c|}
\hline Patología & $\begin{array}{c}\text { Total de caballos } \\
\text { afectados }\end{array}$ & $\begin{array}{l}\text { Porcentaje } \\
\quad(\%)\end{array}$ & $\begin{array}{l}\text { Total machos } \\
\quad(\mathrm{n}=55)\end{array}$ & $\begin{array}{l}\text { Porcentaje } \\
\quad(\%)\end{array}$ & $\begin{array}{l}\text { Total hembras } \\
\qquad(\mathrm{n}=22)\end{array}$ & $\begin{array}{l}\text { Porcentaje } \\
\qquad \%)\end{array}$ \\
\hline Ciego & 19 & 24,67 & $3^{\mathrm{a}}$ & 5,45 & $16^{\mathrm{b}}$ & 72,73 \\
\hline Tártaro & 12 & 15,58 & 10 & 18,18 & 2 & 9,09 \\
\hline Crecimiento excesivo & 5 & 6,49 & 5 & 9,09 & 0 & 0 \\
\hline Enfermedad periodontal & 4 & 5,19 & 4 & 7,27 & 0 & 0 \\
\hline Fractura & 2 & 2,59 & 2 & 3,64 & 0 & 0 \\
\hline Desplazamiento & 1 & 1,3 & 1 & 1,82 & 0 & 0 \\
\hline
\end{tabular}

$\mathrm{N}=77$

Las letras a y b indican diferencia estadística $(\mathrm{P}<0,05)$. 
Arenas 2003, Núñez y col 2004, Estrada 2006, Espinosa 2007, Pimentel y col 2007, Esturillo 2008). Esta baja frecuencia podría ser una característica racial del caballo chileno, ya que en el presente estudio de acuerdo a lo informado por los responsables de los caballos no se habían hecho extracciones de dientes de lobo como ocurre en otros estudios. Las extracciones se basan según Easley (2004) y Scrutchfield (2006) en un concepto errado, en que se cree que el diente de lobo maxilar provocaría molestias al maniobrar el freno, sin embargo, como fue demostrado por Scoggins (2001), el freno se contacta sólo con la mandíbula. Por otra parte, Engelke y Gasse (2003) señalan que las molestias estarían más bien asociadas al traumatismo provocado por el freno en la encía mandibular o lengua, sobre todo si éste es de un tamaño mayor al adecuado; también como indica Linkous (2005) podría observarse molestia en casos de dientes de lobo extremadamente largos. Por otro lado, una de las razones que podría explicar el bajo porcentaje de diente de lobo es que el estudio se realizó sólo en caballos de 5 años o más, ya que se han visto en otros estudios frecuencias mayores en individuos de 1 a 2 años, las cuales tienden a disminuir después de los 3 años por pérdida de este diente al momento de la muda del segundo premolar (Engelke y Gasse 2003). Trece machos (21,3\% de los machos) y tres hembras (7,69\% de las hembras) del total de caballos examinados poseían dientes de lobo, no existiendo diferencia estadística. Tanto en machos como en hembras la frecuencia por sexo es inferior a lo observado en otros trabajos chilenos que señalan que la presencia de diente de lobo en machos era de 26,5 a 73,6\% y en hembras de 20,3 a 84,6\% (Arenas 2003, Espinosa 2007). Todos los dientes de lobo encontrados en este estudio eran maxilares (105-205), coincidente con lo reportado por otros autores (Brigham y Duncanson 2000, Arenas 2003, Estrada 2006, Linkous 2006, Esturillo 2008). Existen estudios que reportan dientes de lobo mandibulares en el 0,4 a 5\% de los caballos (Scrutchfield 2002, Espinosa 2007, Pimentel y col 2007), existiendo predisposición racial, particularmente en standardbred como señala Scrutchfield (2002). La única patología que se presentó en el diente de lobo fue enfermedad periodontal en una pieza 205 , en un caballo (6,25\% del total de caballos con diente de lobo), patología no reportada en otros estudios, en donde si hay reportes de desplazamientos (Dixon y col 1999, Arenas 2003, Estrada 2006) en porcentajes de 0,5 a 5,33\% o dientes supernumerarios (Arenas 2003, Dixon y col 2005). No se encontraron diferencias estadísticas para las diferentes patologías halladas en los segmentos estudiados asociadas a la edad. Finalmente, se puede concluir que en el caballo chileno la frecuencia de patologías dentales en los segmentos estudiados es muy alta, principalmente en incisivos, siendo en general la mayoría de tipo adquirida. Además la baja incidencia de diente de lobo en ambos sexos y la alta incidencia de caninos en ambos sexos, principalmente en hembras, requiere de un estudio mayor para definirlos como una característica racial.

\section{RESUMEN}

Las patologías dentales han ido tomando mayor relevancia clínico veterinaria, aumentando el número de consultas, tratamientos y procedimientos preventivos. En caballos chilenos, la información existente de patologías dentales es nula, por lo que se hace primordial la investigación y recolección de datos en esta raza. Se analizaron 100 caballos pertenecientes a la Asociación de Rodeo Concepción, entre los meses de junio y septiembre del año 2007. Los materiales que se utilizaron fueron puro, linterna, sonda odontológica y espejo odontológico. El examen incluyó reseña, anamnesis y examen dental, registrando todo lo encontrado en una ficha odontológica. Ochenta y ocho ejemplares presentaron algún tipo de patología dental (88\%). Las patologías más frecuentes fueron fracturas con $46 \%$, tártaro con $33 \%$ y braquignatia con $32 \%$, todos estos ubicados en los incisivos ('01-'03). Sesenta y siete caballos ( 55 machos y 22 hembras) poseían uno o más caninos (77\%). De éstos, 38 (49,35\%) presentaba patologías en caninos, siendo lo más frecuente caninos ciegos $(24,67 \%)$. Se encontraron 16 equinos (13 machos y 3 hembras) con uno o más primeros premolares (diente de lobo) (16\%), ninguno mandibular. Sólo se encontró un caso de patología en un primer premolar que correspondió a enfermedad periodontal. Se encontraron diferencias estadísticas asociadas al sexo, para boca en declive en incisivos y caninos ciegos.

\section{AGRADECIMIENTOS}

Al Dr. Mario Briones, del Departamento de Ciencias Pecuarias, Facultad de Ciencias Veterinarias, Universidad de Concepción, por su apoyo en el análisis estadístico de los datos.

\section{REFERENCIAS}

Arenas B. 2003. Estudio de prevalencia de las principales alteraciones dentales de equinos en piezas de matadero. Memoria de título. Escuela de Medicina Veterinaria, Universidad Santo Tomás, Santiago, Chile.

Brigham E, G Duncanson. 2000. Case study of 100 horses presented to an equine dental technician in the UK. Equine Vet Educ 12, 63-67.

Caldwell L. 2006 . A review of diagnosis, treatment, and sequelae of incisor-luxacion fractures in horses (from a dentist's viewpoint). Proceeding American Association of Equine Practitioners 52, 559-564.

Caldwell L. 2006 ${ }^{\text {b }}$. Canine teeth in the equine patient - The guide to eruption, extraction, reduction and others things you need to know. Focus Meeting. American Association of Equine Practitioners, Indianapolis, USA.

Chabchoub A, F Landolsi, M Ben Ali, K Jones, MA Abrougui. 2005. Contribution à l'étude des dominantes en pathologie bucco-dentaire chez les équidés de travail en Tunisie. Reveu Méd Vét 156, 316-322.

Dacre K. 2006. Applied equine dental development. Focus Meeting. American Association of Equine Practitioners, Indianapolis, USA.

Dixon PM. 2003. The etiology, diagnosis and current therapy of developmental and acquired equine dental disorders. $8^{\text {th }}$ Congress on Equine Medicine and Surgery, Geneva, Switzerland.

Dixon PM, I Dacre. 2005. A review of equine dental disorders. Vet J 169, 165-187.

Dixon PM, J Easley, A Ekmann. 2005. Supernumerary teeth in the horse. Clin Tech Equine Pract 4, 155-161.

Dixon PM, WH Tremaine, K Pickles, L Kuhns, C Hawe, J McCann, B McGorum, D Railton, S Brammer. 1999. Equine dental disease part 1: A long term study of 400 cases: disorders of incisor, canine and first premolar teeth. Equine Vet J 31, 369-377. 
Easley J. 2002. Examen dental y oral. En: Baker GJ, Easley J (eds). Odontología Equina. Editorial Inter-médica. Buenos Aires, Argentina, Pp 121-142.

Easley J. 2004. Equine canine and first premolar (wolf) teeth. 50th Proceeding American Association of Equine Practitioners, Denver, Colorado, USA.

Easley J. 2006. Equine dental developmental abnormalities. Focus Meeting. American Association of Equine Practitioners, Indianapolis, USA.

Engelke E, H Gasse. 2003. An anatomical study of the rostral part of the equine oral cavity with respect to position and size of a snaffle bit. Equine Vet Educ 15, 158-163.

Espinosa A. 2007. Identificación y frecuencia de patologías y alteraciones dentales en equinos de matadero de la ciudad de Chillán VIII Región, Chile. Memoria de título, Facultad de Ciencias Veterinarias, Universidad de Concepción, Chile.

Estrada C. 2006. Determinación de desórdenes dentales en equinos de matadero en la Provincia de Concepción, Región del Bío-Bío. Memoria de título, Facultad de Ciencias Veterinarias, Universidad de Concepción, Concepción, Chile.

Esturillo T. 2008. Identificación y frecuencia de desórdenes dentales en caballos Fina Sangre Inglés residentes en el Club Hípico de Concepción. Memoria de título, Facultad de Ciencias Veterinarias, Universidad de Concepción, Concepción, Chile.

Johnson TJ, CM Porter. 2006. Common disorders of incisor teeth and treatment. Focus Meeting. American Association of Equine Practitioners, Indianapolis, USA.

Klugh D. 2006. A review of equine periodontal disease. Proceeding American Association of Equine Practitioners 52, 551-558.

Linkous MB. 2005. Performance dentistry and equilibration. Clin Tech Equine Pract 4, 124-134.
Linkous MB. 2006. Dental conditions affecting the juvenile performance horse (2-5 years). Focus Meeting. American Association of Equine Practitioners, Indianapolis, USA.

Lowder M. 1998. Current nomenclature for the equine dental arcade. Vet Med 93, 754-755.

Núñez C, L Vits, O Araya. 2004. Identificación y prevalencia de patologías dentales en caballos carretoneros de la ciudad de Valdivia. Resúmenes XIII Congreso Chileno de Medicina Veterinaria, Valdivia, Chile.

Peters J, B de Boer, G Broeze-ten Voorde, J Broeze, P Wiemer, T Sterk, T Spoormakers. 2006. Survey of common dental abnormalties in 483 horses in Netherlands. Focus Meeting. American Association of Equine Practitioners, Indianapolis, USA.

Pimentel LFRO, A Zoppa, GES Alves, RF Amaral. 2007. Equine dental disorders: review of 607 cases. Pesq Vet Bras 27 (supl.), 109-110.

Razman, PHL, PM Dixon, SA Kempson, PD Rossdale. 2001. Dental dysplasia and oligodontia in a thoroughbred colt. Equine Vet J 33, 99-104.

Schumacher J. 2006. The equine temporomandibular joint. Focus Meeting. American Association of Equine Practitioners, Indianapolis, USA.

Scoggins RD. 2001. Bits, bitting, and dentistry. Proceeding American Association of Equine Practitioners 47, 138-141.

Scrutchfield WL. 2002. Profilaxis dental. En: Baker GJ, J Easley (eds). Odontología Equina. Editorial Inter-médica. Buenos Aires, Argentina, Pp 205-228.

Scrutchfield WL. 2006. Wolf teeth: How to safely and affectivity extract and is it necessary. Focus Meeting. American Association of Equine Practitioners, Indianapolis, USA.

Tremaine WH. 2004. Diastema and periodontal disease in the horse. Equine Vet Educ 16, 192-193.

Vlaminck L, P Desmet, M Steenhaut, A De Moor. 2001. Dental disease in the horse: a survey based on 283 equine cadaver heads. Vet Surg Abstr 30, 302. 